\title{
A Knowledge Based Analysis on Big Data Analytics in Optimizing Electronic Medical Records in Private Hospitals
}

\author{
https://doi.org/10.3991/ijoe.v17i12.27523
}

Atif M. Gattan $($ 四)

King Abdulaziz University, Jeddah, Saudi Arabia

amgattan@kau.edu.sa

\begin{abstract}
The current examination is to break down the information dependent on the Big Data Analytics in enhancing electronic medical records in clinics and wellbeing focuses. Numerous emergency clinics and medical services habitats are experiencing incapable utilization of enormous information investigation in streamlining electronic medical records (EMRs) to create great bits of knowledge for their clinical practices. Hierarchical conduct and the exercises associated with the medical field are the principal factors in improving the utilization of Big Data Analytics in EMRs. The examination centres around exploring the how the models and methods of information can accomplish enormous information life cycle in EMRs. The examination likewise uncovers the information put together investigation with respect to EMRs use and capacity that assists with improving the significant usage of huge information rehearses. The investigation contributes on advanced wellbeing rehearses by investigating the appropriate adaption of scientific instruments to EMRs to shape the significant utilization of large information examination with EMRs.
\end{abstract}

Keywords_-big data analytics, medical records, hospitals, health care centres etc

\section{Introduction}

Big data analytics advances have shown their guarantee in improving numerous territories of care, from medical imaging and ongoing sickness the board to populace wellbeing and exactness medication. These calculations could build the productivity of care conveyance, lessen managerial weights, and speed up infection determination. Regardless of the relative multitude of good these devices might actually accomplish, the damage these calculations could cause is close to as incredible [1]. Worries about data access and assortment, understood and unequivocal predisposition, and issues with patient and supplier trust in analytics advancements have upset the utilization of these devices in regular medical conveyance. The analysts and associations are attempting to 
discover answers for these issues, encouraging the utilization of big data analytics in medical records for better quality and results [2].

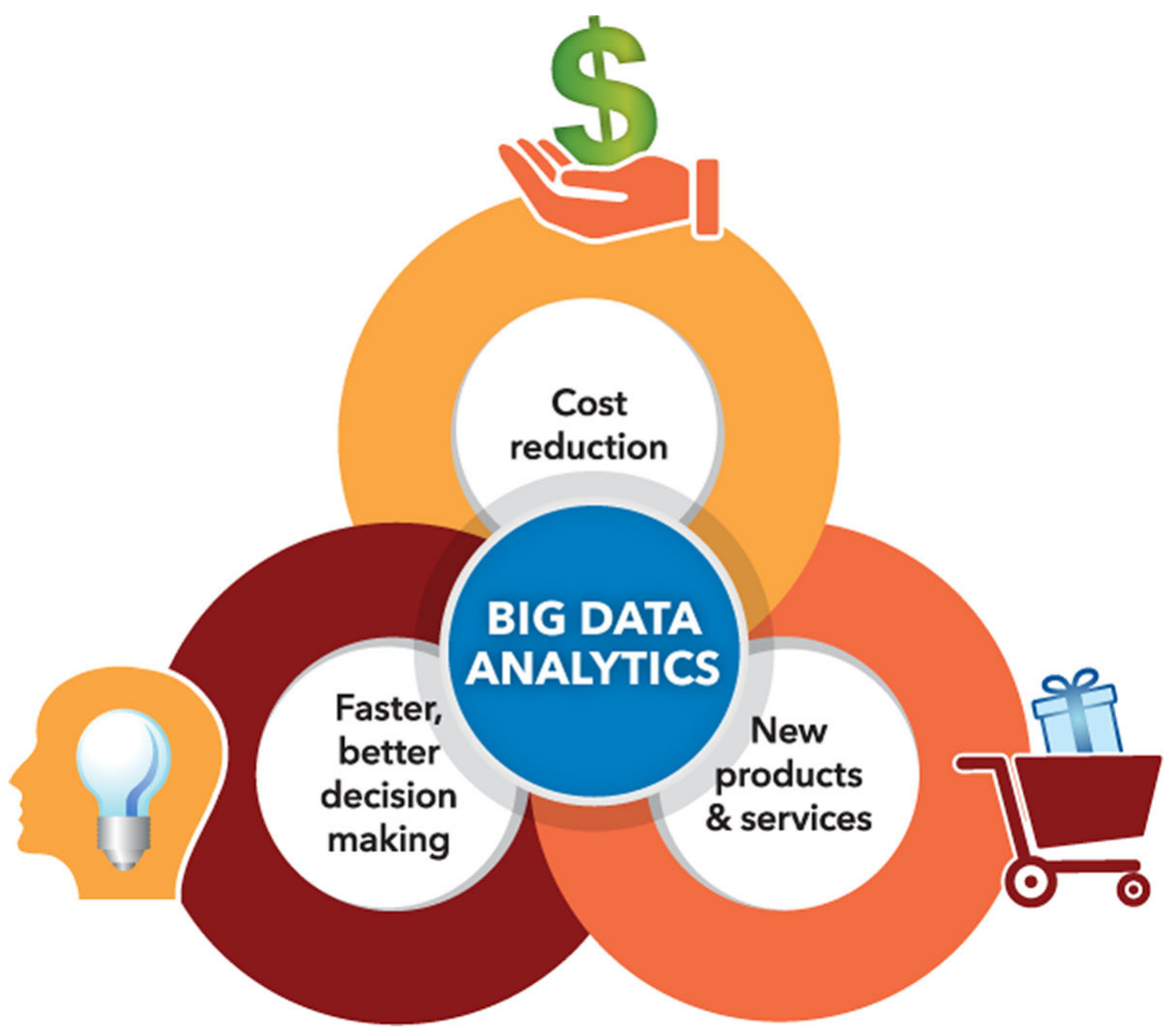

Fig. 1. Big Data Analytics [1]

Figure 1 illustrates how the big data analytics are used to accumulate the satisfactory administrations and handle the patients, clinics and medical care members of the family with countless countries have planned diverse representations of medical offerings information frameworks. These representations for personalized, prescient, sharing and protecting medicine rely on utilizing of electronic medical documents (EMRs) and massive measures of multipart biomedical info and sophisticated grade-omics information. Contemporarily genomics and submit genomics advances yield huge measures of crude knowledge about tricky biochemical and administrative cycles within the living creatures [2-3]. These-omics information is heterogeneous, and at all times they're put away in various knowledge designs. Like these-omics knowledge, the EMRs data are likewise in heterogeneous configurations.

The EMRs information will also be organized, semi-equipped or unstructured; discrete or nonstop. enormous data in medical care and medicine alludes to these different big and difficult knowledge, which they're rough to examine and make do with conventional programming or equipment. enormous data analytics covers reconciliation 
of heterogeneous information, data first-class manipulate, investigation, exhibiting, understanding and approval [4]. Utilization of tremendous information analytics gives exhaustive understanding finding from the obtainable big measure of data. specially, colossal data analytics in remedy and hospital therapy empowers examination of the giant datasets from a large quantity of sufferers, distinguishing businesses and relationship between datasets, simply as developing prescient units utilising data mining methods. huge information analytics in medicine and hospital treatment contains examination of some logical territories, for example, bioinformatics, medical imaging, sensor informatics, scientific informatics and wellness informatics.

To point of improving nature of care through the significant utilization of electronic medical records (EMRs), the public authority has declared the Electronic Medical Record Design and Data Standard in 2018 as a guide for the clinics. In this guide, EMRs are characterized as "a total assortment of advanced medical data recording the medical care delivered to a person in the EMR Standard"[5]. More than twenty years, EMRs has been proposed to upgrade the medical assistance productivity and viability, yet it doesn't imply that just embracing the EMRs framework could prompt those advantages. Medical care suppliers need to make the EMR a daily practice in the dayby-day work framework to understand the recompense. In this manner, Wellbeing Data Innovation for Financial and Clinical Wellbeing (HITECH) Act presents the "significant use" of EMR as the objective of appropriation [3]. The principal objective of Act is to make significant and helpful computerized medical records, including the passage and capacity of EMRs, and upgrade the use of EMRs.

Starting at 2019, medical data had arrived at 250 exabytes $(1 \mathrm{~EB}=1018$ bytes $)$ around the world, for the most part as EMRs. However, significant vulnerability actually stays about the utilization of big data analytics inside EMRs and its effect on medical execution. Such battles are because of not just deficient asset and one-sided asset portion at the public level yet additionally absence of preparation and administration for the utilization of big data analytics inside EMRs at the medical clinic level [3]. To address this test, albeit numerous emergency clinics/heath care focuses have contributed a lot of cost, time and assets in learning the execution and usage of EMRs, they are as yet experiencing ineffectual utilization of big data analytics inside EMRs to produce great data for dynamic and decrease wellbeing differences. One of the vital purposes behind this trouble is the absence of full thought of EMRs wellness to the particular circumstances of the specific association. pay greater attention to understand how to absorb the diverse knowledge of EHRs. As such, little attention has been paid to understanding the role of knowledge mode in improving the use of big data analytics within EHRs. In this study, thus, we examine the relationship between the knowledge about big data analytics within EHRs and the outcome of EHRs adoption (i.e., meaningful use of EHRs). The remainder of this paper is structured as follows: the next section serves as our theoretical background, which leads to the development of the research model and associated hypothesis; followed by our research method, findings and discussions, contributions to research, implications for practice and recommendations, then limitations and future research directions are discussed as our conclusion. It is significant for medical services experts to focus more on see how to retain the different information on EMRs. Accordingly, little consideration has been paid to understanding the part of information mode in improving the utilization of big data analytics inside EMRs. 
In this investigation, consequently, we analyse the connection between the information about big data analytics inside EMRs and the result of EMRs selection (i.e., significant utilization of EMRs) [6].

\section{$2 \quad$ Big data characteristics}

The time period enormous information is portrayed with the aid of the accompanying attributes: esteem, volume, velocity, assortment, veracity and inconstancy, supposed as 6 "Versus". as opposed to these 6 "Versus", a couple of creators have characterised greater than these 6 houses to portray significant information qualities.

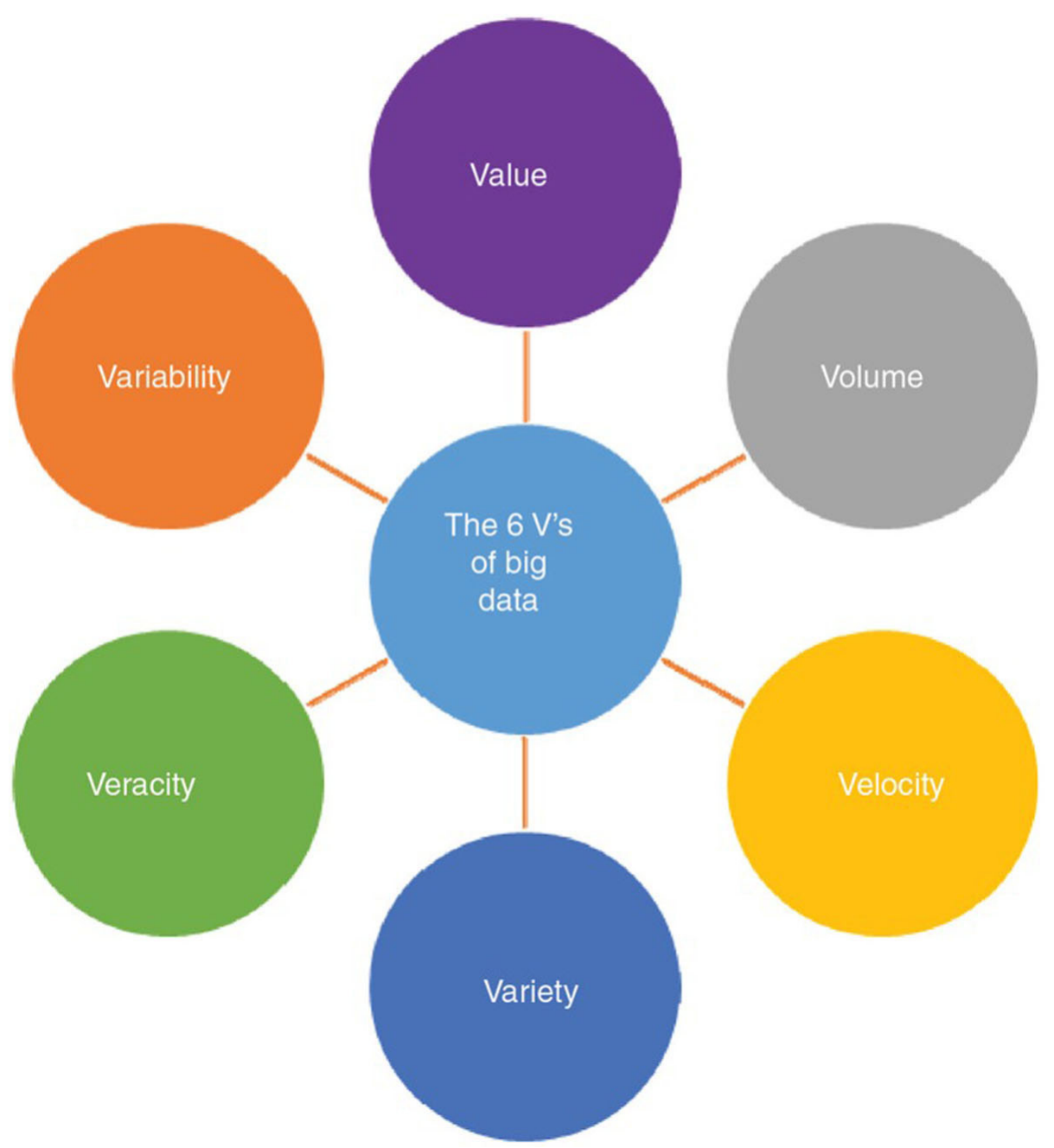

Fig. 2. 6 V's of big data 
Figure 2 show the capacity of wellness and clinical data is relied upon to bring strongly up in the years ahead, commonly estimated in terabytes, petabytes even yottabytes. quantity alludes to the measure of knowledge, even as pace alludes to data relocating just as and to the velocity and recurrence of data creation, making ready and investigation. Intricacy and heterogeneity of countless datasets, which will also be equipped, semi-geared up and unstructured, allude to the assortment [7]. Veracity referrers to the info excellent, significance, vulnerability, dependability and prescient valued at, whilst fluctuation respects about consistency of the data after a while. The estimation of the giant information alludes to their sound examination, which need to be main to the patients and clinicians. fascinated about the large knowledge features, data looking, stockpiling and investigation, an peculiarly becoming and promising programming stage for advancement of utilizations that can deal with big data in medication and medical care is the open-supply dispersed data making ready stage Apache Hadoop MapReduce that is dependent upon data-serious registering and NoSQL knowledge demonstrating methods [8].

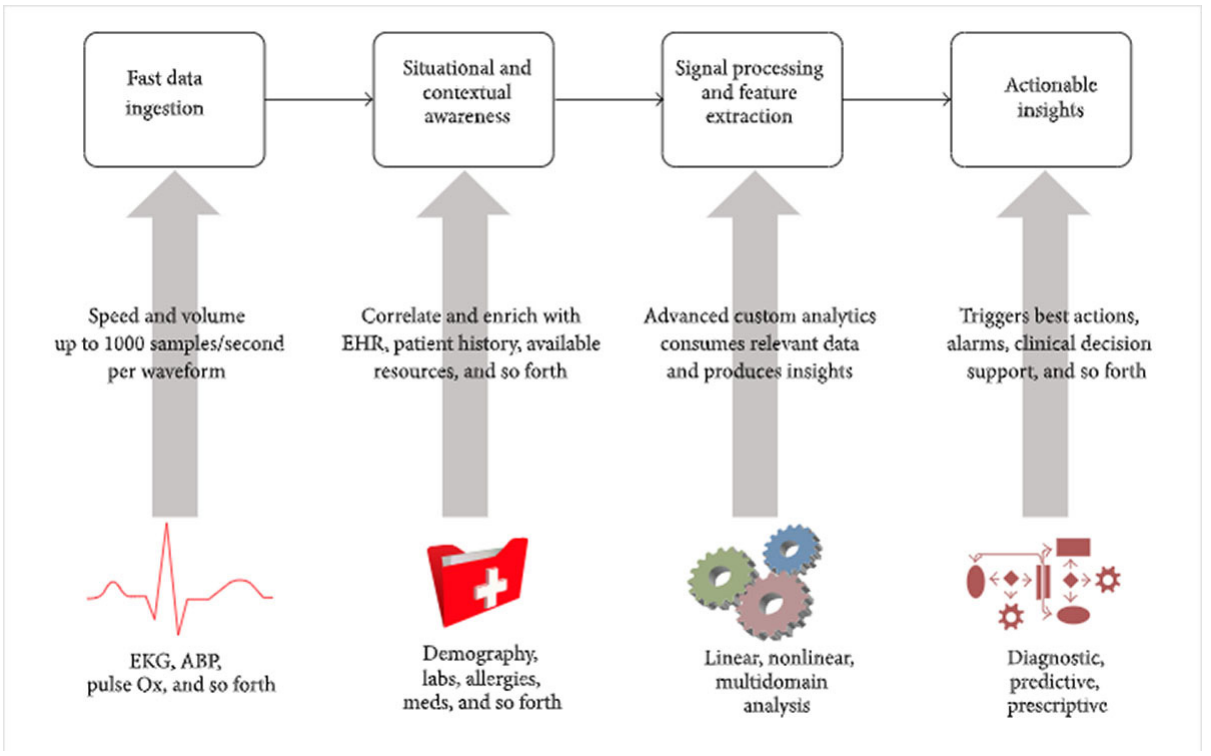

Fig. 3. Representation of big data flow [2]

Figure 3-Fascinated with the huge knowledge characteristics, data watching, stockpiling and investigation, a particularly fitting and promising programming stage for advancement of utilizations that may take care of colossal data in medication and hospital therapy is the open-supply dispersed data preparing stage Apache Hadoop MapReduce that is determined by information-serious registering and NoSQL information demonstrating ways. 


\section{Optimizing the electronic medical records through big data analytics}

The significant utilization of EMRs is critical for improving clinical tasks and medical care administration. Big data analytics is an apparatus that empowers medical services associations to arrive at this objective by enhancing EMRs through scientific calculations. In this manner they can give medical services benefits all the more productively and precisely, streamline existing activities, and forestall some medical dangers [9]. Undoubtedly, the utilization of big data analytics inside EMRs is established in the idea of data life cycle structure that comprises of three parts: data assortment, data stockpiling, and data use. These coherent parts that perform explicit capacities empower medical expert to see how to change the EMRs into significant medical experiences through big data analytics.

Data assortment. This segment contains all the data sources and substance kind of EMRs. By and large, The EMRs are isolated into organized data (e.g., patient socioeconomics, prescription history, wellbeing status and lab results) and unstructured data (e.g., finding notes, medical designs, and medical pictures). These data are gathered from different medical units inside the clinic or from outer units.

Data storage.The EMRs are put away into fitting databases relying upon the wellspring of data and substance design. This part expects to deal with data from the different data sources by two stages: change and capacity. The change motor is fit for moving, cleaning, parting, interpreting, combining, arranging, and approving EMRs. This part presents the proposed fraud detection model and depict its stages, as in Figure 5. The model is divided into 3 phases to be specific input stage, during applying the model stage and output stage.

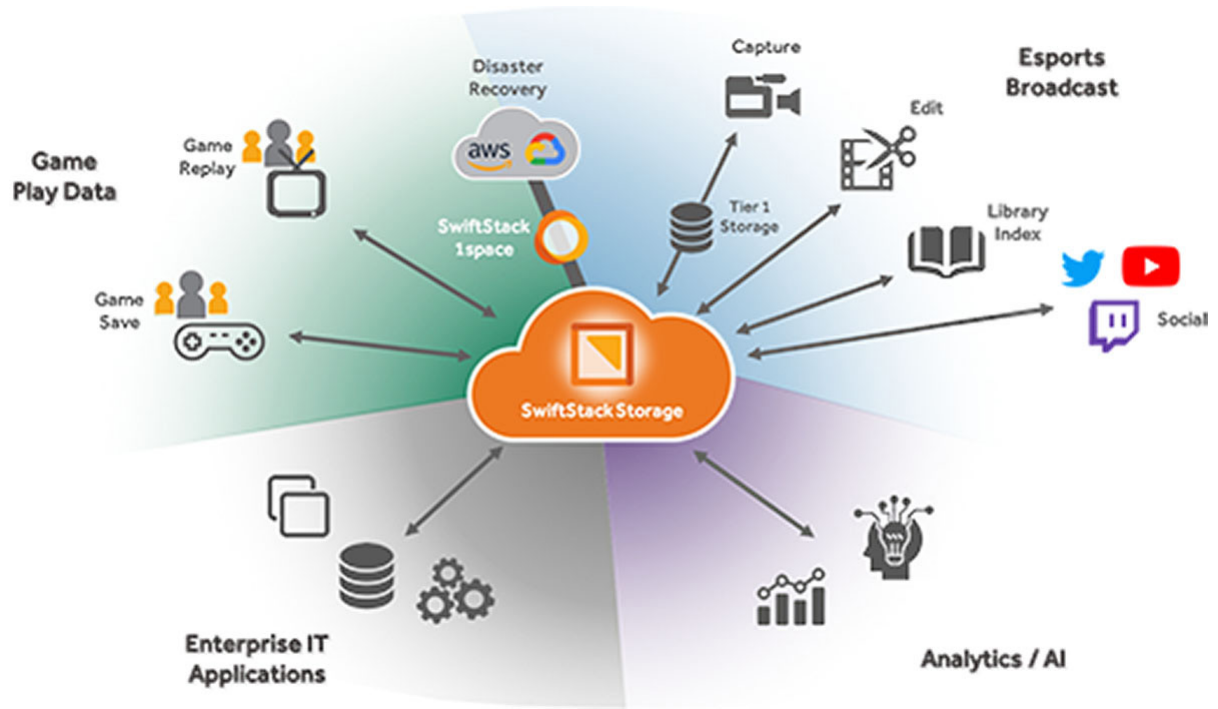

Fig. 4. Data store representation [2] 
Figure 4, is an example, organized EMRs data will be separated from medical care data frameworks and changed over into a particular standard data design, arranged by the model (e.g., patient character, medical status medicine history), and afterward the record in the opportune spot. In the subsequent stage, the EMRs are stacked into the objective databases (e.g., Database The board Framework; DBMS, Hadoop dispersed document frameworks; HDFS, or in a cloud) for additional investigation.

Data utilization. This component is used to process all kinds of EHRs and report the summarized results for clinical decision making. The analysis of EHRs includes Map/ Reduce, stream computing, and in-database analytics, depending on the type of data and the purpose of the analysis. Map/Reduce can provide the ability to process massive unstructured and structured EHRs in batch form in a massively parallel processing environment. Stream computing can support near real time or real time analysis for EHRs. Though stream computing, medical staffs can track EHRs in motion in order to respond to unexpected events and determine next-best actions. In-database analytics is commonly used data mining approach that allows EHRs to be analyzed within database. It can provide high-speed parallel processing and offer a safe environment to process confidential patient information. This component also generates various visualization reporting and real-time and meaningful business insights derived from the analysis. The reporting system is a critical big data analtyics feature that allows EHRs to be visualized in a meaningful way to support medical staff day-to-day operations and clinical decisions.

Data utilization. This part is utilized to deal with a wide range of EMRs and report the summed-up outcomes for medical dynamic. The investigation of EMRs incorporates Guide/Diminish, stream registering, and in-database analytics, contingent upon the kind of data and the motivation behind the examination.

Guide/Lessen can give the capacity to handle enormous unstructured and organized EMRs in group structure in a hugely equal preparing climate. Stream figuring can uphold close to ongoing or continuous examination for EMRs [3]. In spite of the fact that stream figuring, medical staffs can follow EMRs moving to react to startling occasions and decide next-best activities. In-database analytics is usually utilized data mining approach that permits.

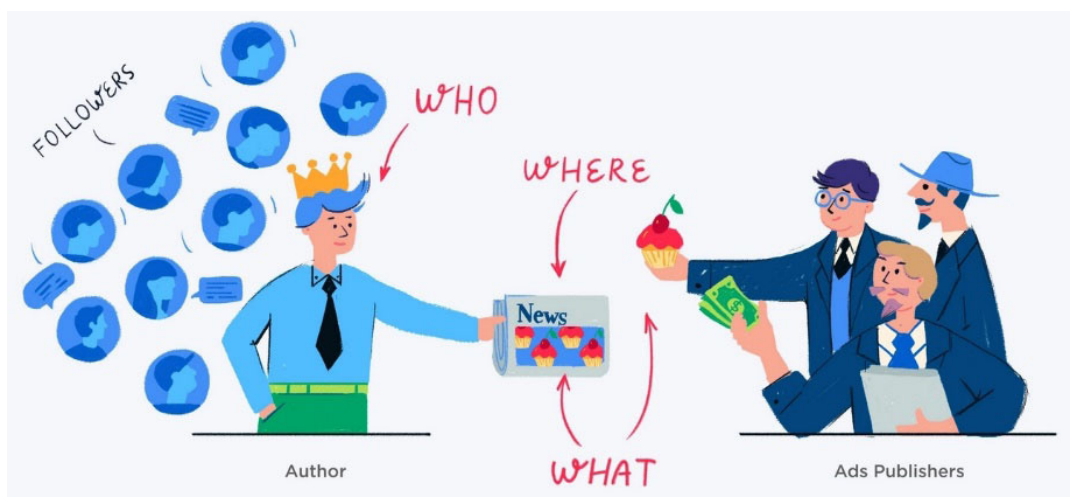

Fig. 5. Data utilization in big data [3] 
Figure 5 shows how EMRs is to be investigated inside database. It can give rapid equal preparing and offer a protected climate to handle classified patient data. This part additionally creates different representation announcing and continuous and significant business bits of knowledge got from the examination. The announcing framework is basic big data analytics highlight that permits EMRs to be pictured in a significant manner to help medical staff everyday activities and medical choices.

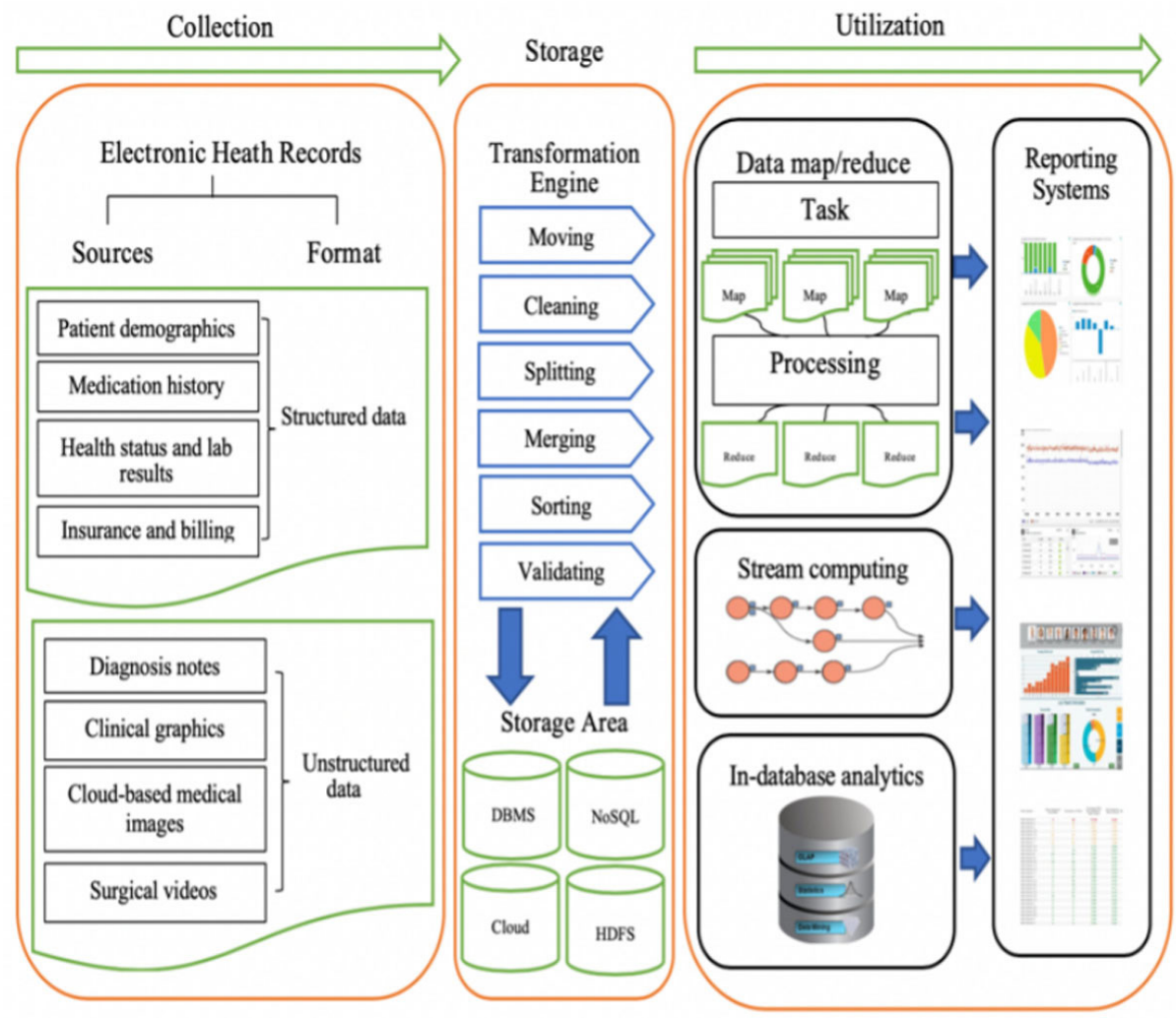

Fig. 6. Process of big data in medical record maintenance [3]

Figure 6 exhibits the medical services are a multi-dimensional framework set up with the sole focus on the avoidance, analysis, and therapy of wellbeing related issues or debilitations in people. The significant segments of a medical care framework are the wellbeing experts (doctors or attendants), wellbeing offices (centres, emergency clinics for conveying prescriptions and other finding or therapy innovations), and a financing foundation supporting the previous two. The wellbeing experts have a place with different wellbeing areas like dentistry, medication, birthing assistance, nursing, brain science, physiotherapy, and numerous others. Medical care is needed at a few levels relying upon the desperation of circumstance (Chen, M)2. Experts serve it as the main mark of meeting (for essential consideration), intense consideration requiring talented experts (auxiliary consideration), progressed medical examination and therapy 
(tertiary consideration) and profoundly phenomenal demonstrative or surgeries (quaternary consideration).

At all these phases, the wellness professionals are dependable for more than a few forms of knowledge like sufferer's clinical history (analysis and solutions associated information), medical and scientific data (like data from tomography and study facility assessments), and other remoted or character scientific data. Already, the typical activity to retailer such scientific records for a patient used to be as either written by using hand notes or composed stories. indeed, even the outcomes from a clinical evaluation had been put away in a paper record framework. certainly, this coaching is really historic, with the most headquartered case reviews existing on a papyrus text from unfamiliar international locations that traces all the approach posterior to $1600 \mathrm{BC}$.

In Stanley Reiser's words, the medical case money owed freeze the scene of sickness as a narrative where patient, household and the specialist are a section of the plot". With the looks of computer frameworks and its latent capability, the digitization of each single clinical test and scientific records within the medical services frameworks has come to be a norm and greatly embraced follow in this day and age [3]. In 2003, a division of the public Foundations of Sciences, Designing, and medicine referred to as group of remedy picked the expression "electronic scientific files" to address documents stored up for bettering the hospital treatment subject in the direction of the skills of sufferers and clinicians. digital scientific records (EMR) as characterised through Murphy, Hanken and Waters are computerized scientific documents for sufferers any knowledge picking out with the prior, gift or future bodily/psychological wellbeing or state of a character which lives in digital approach(s) used to capture, keep in touch, get, store, get well, connect and manage media knowledge for the elemental function of giving clinical services and scientific-related administrations".

\section{$4 \quad$ Electronic medical records}

Observe that the public Foundations of wellness (NIH) as of late declared the "we all" exercise that intends to acquire at the least 1,000,000 sufferers' information like EMR, together with medicinal imaging, socio-conduct, and ecological knowledge ended the path of the following no longer a long time. EMRs have presented countless advantages for taking care of current clinical offerings associated data. under, we depict a portion of the trademark benefits of using EMRs [3, 10]. The primary benefit of EMRs is that scientific services specialists have an accelerated admittance to the entire scientific earlier of a patient. the info comprises clinical conclusions, treatments, knowledge recognized with recognized hypersensitivities, socioeconomics, medical debts, and the outcomes received from unique research facility exams.

The acknowledgment and treatment of clinical stipulations for this reason is time productive due to the fact that of a shrink within the slack season of prior scan outcome. With time now we have noticed a primary diminishing within the excess and extra assessments, lost requests and ambiguities caused via indecipherable penmanship, and an improved consideration coordination between more than a few scientific services suppliers. Beating such calculated errors has caused minimize in the wide variety of treatment sensitivities by lessening mistakes in drug portion and recurrence $[2,11]$. 
clinical offerings authorities have likewise found out admittance over on-line and electronic stages to beef up their medical practices just about using programmed updates and prompts with regard to immunizations, unique lab outcome, malignancy screening, and different occasional registration.

There could be a more noteworthy coherence of care and effortless mediations by way of encouraging correspondence amongst numerous hospital therapy suppliers and patients. they can be regarding digital approval and rapid safeguard endorsements for the reason that of much less administrative work. EMRs empower quicker knowledge restoration and motivate announcing of key hospital therapy excellent markers to the associations, and furthermore fortify common wellbeing commentary through immediate revealing of illness flare-ups. EMRs moreover give relevant data in regards to the nature of handle the recipients of consultant medical insurance plan programs and may aid control the expanding costs of wellness care coverage advantages $[3,12]$. At lengthy final, EMRs can minimize or wholly dispense with deferrals and disarray in the charging and claims the executive's zone. The EMRs and net collectively assistance provide admittance to a big quantity of health-related medical knowledge common for patient life.

\section{Digitization of healthcare and big data}

A digital scientific file (EMR) retailers the commonplace medical and medical data amassed from the patients. EHRs, EMRs, character health file (PHR), scientific follow the board programming (MPM), and countless different hospital therapy knowledge segments all things regarded can potentially enhance the pleasant, administration effectiveness, and costs of scientific services alongside the lessen of medical mistakes. The enormous knowledge in clinical services accommodates the hospital treatment payer-supplier data (like EMRs, drug store relief, and protection files) alongside the genomics-pushed analyses, (for example, genotyping, high-quality articulation knowledge) and different information got from the eager snare of net of matters (IoT) [3]. The reception of EMRs was delayed toward the start of the 21 st century anyway it has grown to be considerably after 2009. Figure 7 illustrates the administration and utilization of such medical care data has been regularly area to knowledge innovation. The turn of events and utilization of wellness staring at objects and related programming that can create cautions and present the health associated information of a sufferer with the distinctive medical care suppliers has got force, principally in constructing up a consistent biomedical and health checking framework. These objects are producing a massive measure of knowledge that can be dissected to offer regular clinical or medical consideration. The utilization of gigantic information from hospital treatment shows ensures for bettering health results and controlling costs. 


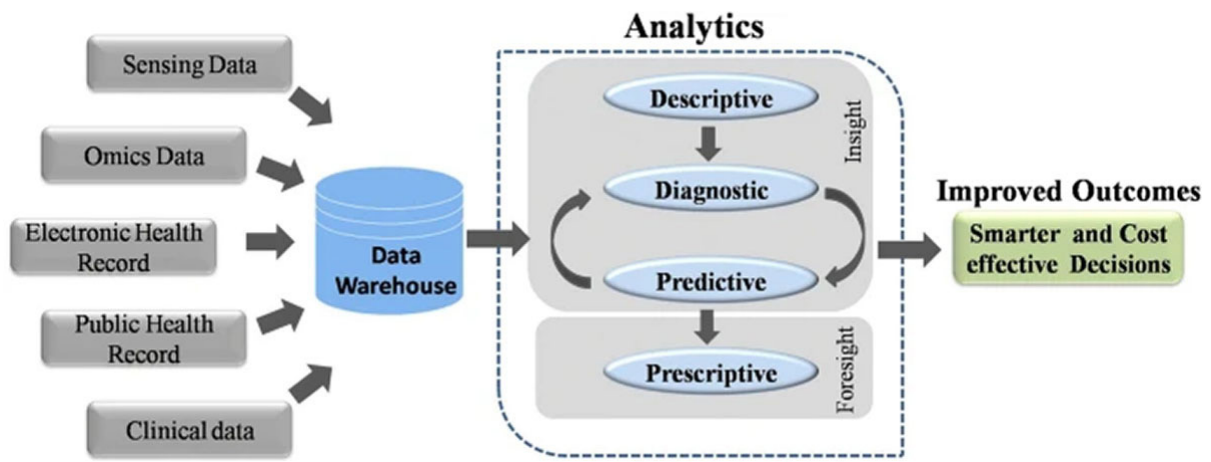

Fig. 7. Big data digitalization

\section{Challenges in big data analytics}

Utilizations of massive data analytics can increase the sufferer-cantered help, to recognize distribution infections prior, produce new experiences into health problem programs, reveal the nature of the scientific and scientific offerings foundations simply as give higher remedy systems data mining methods utilized on EMRs, web and on-line media knowledge empower recognizing the ideal affordable principles within the clinics, distinguishing the affiliation ideas in the EMRs and uncovering the sickness gazing and health-based patterns. additionally, reconciliation and investigation of the information with quite a lot of nature, like social and logical, can immediate new information and expertise, investigating new idea, recognizing included up designs this present day, evolved cells are mighty levels to carry individual messages to patients to incorporate them in conduct changes to fortify their prosperity and medical problem [13].

The cell phone messages can replacement conveying of scientific and inspirational advices to the sufferers. regarding of tremendous sum information, some tricky disorders have got to be notion of. acquiring excessive-throughput - omics data is attached to the cost of scan estimations. concerning array of the data sources, the commotion of the experiment - omics data and the assortment of the trial methods, ecological conditions, organic nature have to be idea of, before mix of these heterogeneous knowledge and prior to making use of of the data mining techniques. various information mining ways will also be utilized on these heterogeneous biomedical knowledge sets, for illustration, oddity vicinity, grouping, association, affiliation controls just as rundown and illustration of these gigantic knowledge units. These deficiencies may immediate the untrustworthiness of a portion of the information focuses, like lacking features or exceptions. 


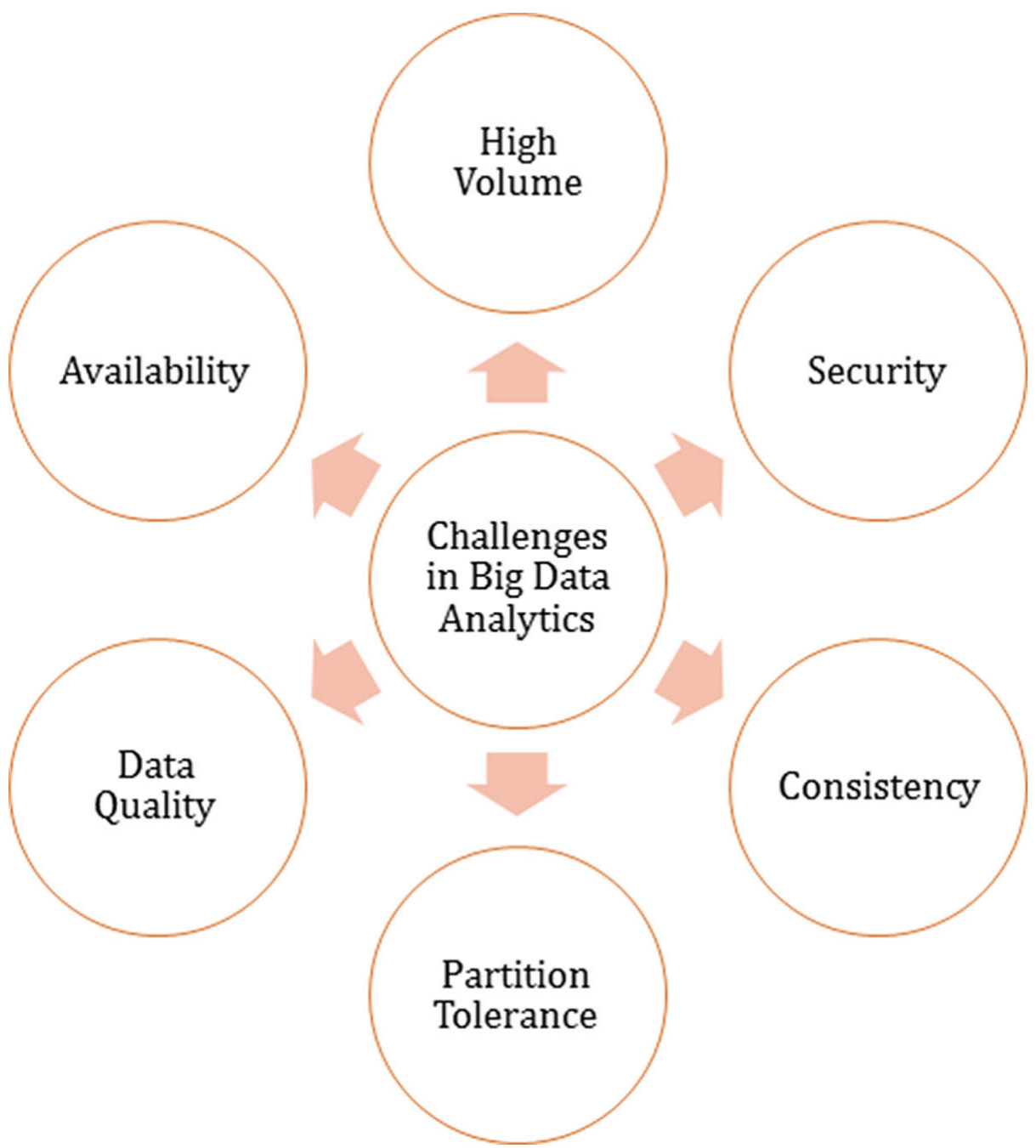

Fig. 8. Big data analytics - challenges

Figure 8, infers the challenges, Regardless of these downsides of the-omics information, EMRs data are particularly plagued by the staff who entered the sufferer's knowledge, which will immediate entering missing characteristics, faulty information considering that of missteps, misconstruing or flawed translation of the first information. combo of information from exclusive databases and normalization for lab conventions esteems actually keep trying out issues [14]. high dimensionality of the-omics data implies, that there have so much a larger number of measurements or highlights than the number of checks, and on the opposite part the EMRs data which recognize to the people/sufferers, makes information mining tactics to be quite 
intricate challenge. The resulting stage is the pre-preparing of the data, which for the most section embody taking care of boisterous data, anomalies, lacking qualities, data change and standardization. this knowledge pre-getting ready empowers to be utilized measurable strategies and data mining strategies and for this reason the gigantic data analytics best and outcome can strengthen and might outcome with discovering of novel understanding. This epic information obtained with the aid of incorporation of the- omics and EMRs information will have to results with enlightening of the performed hospital therapy to the patients too to cutting edge dynamic through the medical services alternative strategy creators [15]. Figure 9 shows big data privacy and security infrastructure.

\section{$7 \quad$ Big data privacy and security}

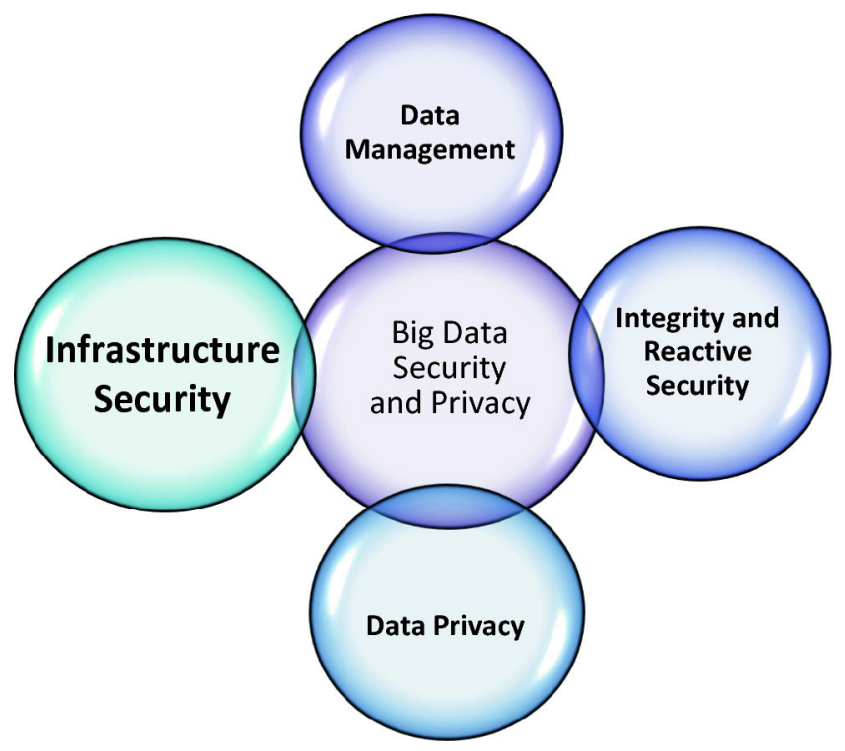

Fig. 9. Big data privacy and security

Two gigantic problems in the direction of large knowledge in hospital therapy and medication are security and safety of the men and women/sufferers. All scientific data are certainly smooth and more than a few nations consider about this information as legitimately controlled through the sufferers. to address these protection and security challenges, the massive information analytics programming preparations will have to make use of progressed encryption calculations and pseudo-anonymization of the man or woman data. These product preparations have got to supply security on the group stage and affirmation for each complicated purchaser, be certain defense and safety, simply as set up exceptional administration ideas and practices. 


\section{Conclusion}

Its significant data analytics in treatment and hospital therapy is totally encouraging cycle of incorporating, investigating and dissecting of giant sum elaborate diverse data with more than a few natures: biomedical data, exploratory information, electronic health files data and online media data. blend of such varied information makes gigantic information analytics to interlace a couple of fields, for instance, bioinformatics, scientific imaging, sensor know-how science, scientific informatics, wellness understanding processing and computational biomedicine [5]. As yet another work, the huge knowledge characteristics provide compatible premise to make use of promising programming levels for development of utilizations that may care for large knowledge in medicine and medical care. One such stage is the open-source conveyed knowledge preparing stage Apache Hadoop MapReduce that utilization enormous equal dealing with (MPP). These applications will have to empower applying data mining approaches to these heterogeneous and complex knowledge to uncover hid examples and novel expertise from the info. Ongoing equipment trends in processor innovation, more present forms of recollections/community design will restrict the time spent in relocating the info from potential to the processor in circulated surroundings.

\section{Future prospects}

These days, different biomedical and medical services apparatuses like genomics, versatile biometric sensors, and cell phone applications create a big measure of data. Subsequently, it is obligatory for us to think about and survey that can be accomplished utilizing this data. For instance, the examination of such data can give further experiences regarding procedural, specialized, medical and different kinds of enhancements in medical services. After a survey of these medical services methodology, apparently the maximum capacity of patient-explicit medical forte or customized medication is in progress. The aggregate big data examination of EMRs and other medical data is consistently helping construct a superior prognostic structure [5]. The organizations offering support for medical care analytics and clinical change are without a doubt contributing towards better and viable result. Shared objectives of these organizations incorporate decreasing expense of analytics, creating successful Clinical Choice Help (Discs) frameworks, giving stages to better treatment systems, and recognizing and forestalling extortion related with big data.

Nevertheless, practically every person of them face difficulties on government problems like how private information is handled, shared and remained careful. The consolidated pool of knowledge from hospital treatment associations and biomedical scientists have precipitated a advanced standpoint, assurance, and remedy of one of a kind diseases [5]. This has additionally helped in building a superior and better custom-made clinical offerings structure. present hospital therapy society has understood the ability of significant knowledge and on this method, have carried out massive knowledge analytics in medical services and scientific practices. Workstations to quantum PCs are serving to in setting apart giant data from enormous knowledge in enormously diminished time-frames. 


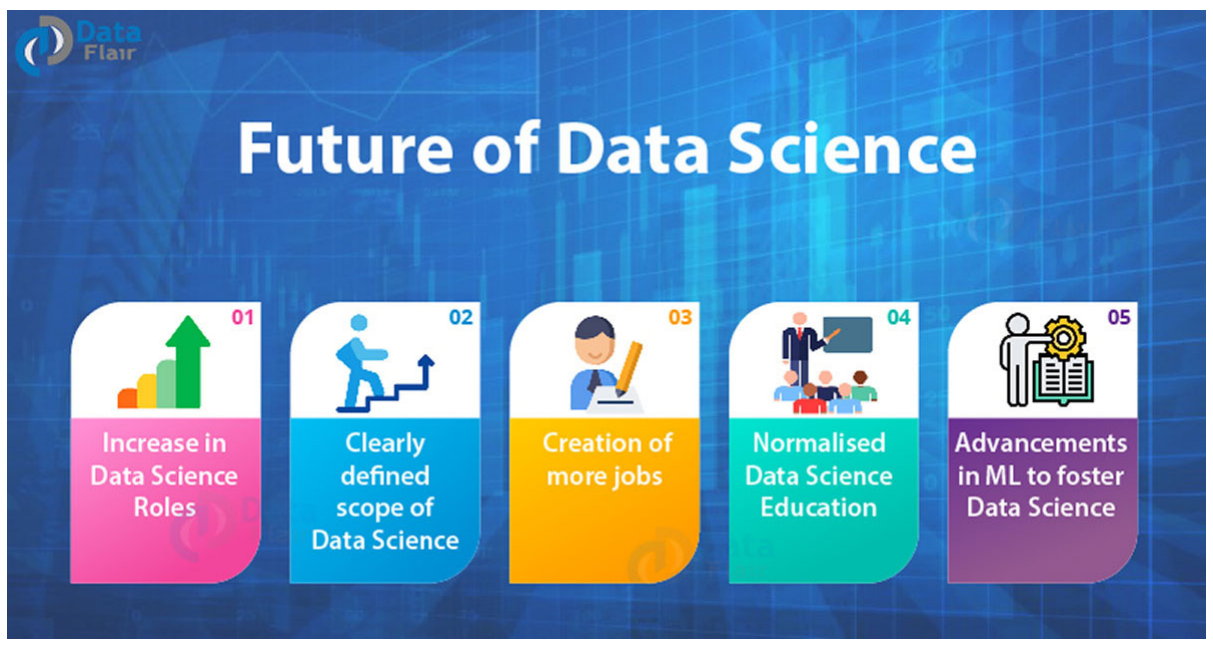

Fig. 10. Future of data science[5]

With excessive any desires for keeping apart new and significant know-how that may give a boost to the current popularity of hospital treatment administrations, analysts are diving into biomedical enormous knowledge however the basis challenges. medical preliminaries, investigation of drug store and protection guarantees together, revelation of biomarkers is a section of a novel and ingenious technique to dissect clinical services enormous knowledge. big knowledge analytics affect the gap within organized and unstructured knowledge sources. The move to an incorporated information local weather is a wonderful concern to outlive. properly intriguing, the regular of giant data intensely depends upon the probability of the more the data, the more experiences you'll gather from this information and can make expectations for future events.

It is legitimately projected through one-of-a-kind responsible counselling organizations and medical care companies that the large knowledge hospital treatment market is competent to advance at a splendid rate. nevertheless, in a constrained capacity to focal point have obvious a variety of analytics at present being used that basically have an impact on the dynamic and execution of hospital therapy industry [5]. The dramatic development of scientific information from extraordinary areas has confined computational professionals to plot innovative methodologies to dissect and decipher such enormous measure of knowledge inside a given time period.

\section{References}

[1] Dash, S., Shakyawar, S. K., Sharma, M., and Kaushik, S. (2019). Big data in healthcare: Management, analysis and future prospects. Journal of Big Data, 6: 54. https://doi. org/10.4018/IJBDAH.2019070103; https://doi.org/10.1186/s40537-019-0217-0

[2] Ristevski, B., and Chen, M. (2018). Big data analytics in medicine and healthcare. Journal of Integrative Bioinformatics, 15(3). https://doi.org/10.1515/jib-2017-0030 
[3] Khanra, S., Dhir, A., Islam, A. K. M. N., and Mäntymäki, M. (2020). Big data analytics in healthcare: A systematic literature review. Enterprise Information Systems, 14: 878-912. https://doi.org/10.1080/17517575.2020.1812005

[4] HealthITAnalytics. (2020, October 2). 4 emerging strategies to advance big data analytics in healthcare. HealthITAnalytics. https://healthitanalytics.com/ news/4-emerging-strategies-to-advance-big-data-analytics-in-healthcare.

[5] Zhang, C., Ma, R., Sun, S., Li, Y., Wang, Y., and Yan, Z. (2019). Optimizing the electronic health records through big data analytics: a knowledge-based view. IEEE Access, 7: 136223-136231. https://doi.org/10.1109/ACCESS.2019.2939158

[6] El-Seoud, S. A., El-Sofany, H. F., Abdelfattah, M., and Mohamed, R. (2017). Big Data and Cloud Computing: Trends and Challenges. International Journal of Interactive Mobile Technologies, 11(2). https://doi.org/10.3991/ijim.v11i2.6561

[7] Shaikh, A., and Rajab, K. D. (2020). Special Issue on New Frontiers in Data Sciences and Data Analytics Tools and Applications. Advances in Data Science and Adaptive Analysis 12(2), 2002001. https://doi.org/10.1142/S2424922X20020015

[8] Liu, Q., and Zeng, L. (2020). Design and Application of Experimental Teaching System Driven by Big Data Technology in Economics and Management Majors. International Journal of Emerging Technologies in Learning (iJET), 15(11), 56-66. https://doi.org/10.3991/ ijet.v15i11.12997

[9] Muis, I., Wonorahardjo, S., and Budiasih, E. (2021). Big Data Support for Problem Solving Method in Mass Spectrometry Topic in Modern Analytical Chemistry Course. International Journal of Interactive Mobile Technologies, 16(9). https://doi.org/10.3991/ijim. $\underline{\mathrm{v} 15 \mathrm{i} 09.21569}$

[10] Shaikh, A., Uddin, M., Elmagzoub, M. A., and Alghamdi, A. (2020). PEMC: Power Efficiency Measurement Calculator to Compute Power Efficiency and $\mathrm{CO}_{2}$ Emissions in Cloud Data Centers. IEEE Access, 8, 195216-195228. https://doi.org/10.1109/ACCESS.2020.3033791

[11] Zhang, X., and Zhang, J. (2018). Complex Big Data Analysis Based on Multi-granularity Generalized Functions. International Journal of Online Engineering, 14(4). https://doi. org/10.3991/ijoe.v14i04.8368

[12] Saeed, S., Shaikh, A., Memon, M. A., and Naqvi, S. M. R. (2018). Impact of data mining techniques to analyze health care data. Journal of Medical Imaging and Health Informatics, 8(4), 682-690. https://doi.org/10.1166/jmihi.2018.2385

[13] Russom, P. (2011). Big data analytics. TDWI best practices report, fourth quarter, 19(4), $1-34$.

[14] Kambatla, K., Kollias, G., Kumar, V., \& Grama, A. (2014). Trends in big data analytics. Journal of parallel and distributed computing, 74(7), 2561-2573. https://doi.org/10.1016/j. jpdc. 2014.01 .003

[15] Shaikh, M., Memon, M.A., Shaikh, A., and Deeba, F. (2016). Analyzing Virtual Machine Live Migration in Application Data Context. INTERNATIONAL JOURNAL OF ADVANCED COMPUTER SCIENCE AND APPLICATIONS, 7(5), 401-408. https://doi.org/10.14569/ IJACSA.2016.070554

\section{Author}

Atif M. Qattan, Ph.D, Associate Professor, my interest subject is distant library survives. I have been published different articles in different journals in Arabic and English language.

Article submitted 2021-09-13. Resubmitted 2021-10-17. Final acceptance 2021-10-19. Final version published as submitted by the authors. 